\title{
Imparare e insegnare non sono la stessa cosa
}

\section{Riccardo Cimaglia}

PUBBLICATO: 15 NOVEMBRE 2019

\section{Quesito:}

Sono pervenute in redazione varie richieste circa i verbi insegnare e imparare, soprattutto riguardo all'uso del verbo imparare come sinonimo di insegnare.

\section{Imparare e insegnare non sono la stessa cosa}

I n italiano standard questi due verbi sono portatori di due distinti significati. Riferendoci al Sabatini-Coletti, s.v., il verbo transitivo insegnare, che richiede l'oggetto diretto della cosa insegnata e loggetto indiretto della persona cui si insegna, indica l'atto di "fornire nozioni teoriche o elementi pratici a qlcu., in modo che apprenda qlco.": Il prof. Bianchi insegna la storia romana ai suoi alunni; in luogo dell'oggetto diretto possiamo trovare una frase infinitiva introdotta da $a$ : L'istruttore ci insegna a nuotare. Sinonimi di insegnare sono istruire, addestrare.

Il verbo imparare, invece, indica l'atto di "acquisire conoscenze o capacità attraverso lo studio, l'esercizio, l'applicazione"; anch'esso è transitivo, richiede cioè l'oggetto diretto della cosa che si impara, e, in aggiunta, il complemento di origine che rappresenta la cosa o la persona da cui si impara: Maria sta imparando il cinese da Alessandro; Imparai molto dalla nostra collaborazione. In luogo dell'oggetto diretto, anche in questo caso, possiamo avere un'infinitiva introdotta da a: Ho imparato a stirare le camicie. Sinonimo di imparare è apprendere.

Sempre il Sabatini-Coletti registra l'uso regionale centro-meridionale (che trova riscontro in moltissimi dialetti, tra cui il napoletano e il romanesco, che usa insegnà(re) nel senso di 'indicare') del verbo imparare con il valore di "insegnare qlco. a qlcu., specialmente con il primo argomento espresso da frase (introdotto da a): ha imparato a scrivere al figlio".

Questa accezione del verbo imparare è attestata anche in testi letterari, a partire dal secolo XVI:

Se il canuto del tempo, che più di quindici lustri gli è suto maestro, non gli ha imparato tal cosa, uno indocile si può chiamare discepolo. (Pietro Aretino, Lettere)

Dubito che abbia potuto trovarsi nella lingua tedesca tutto intero quel poetico che le regole non insegnano e gli studi non imparano. (Pietro Giordani, Volgarizzamento di un discorso della baronessa di Staël: Sulla maniera e la utilità delle traduzioni, $\mathrm{I} 8 \mathrm{I} 6)$

Essa la diva / con le dita d'ambrosia, essa da gli occhi / tergea de la mortal giovine il pianto; / e dolce un canto le imparava. (Giosue Carducci, Juvenilia, LXVII, Maggio e Novembre, vv. 37-40)

Quello là vuol far l'offeso eh? Adesso glielo imparo io: non gli telefono per almeno un mese. (Dino Buzzati, Un amore, 1963)

Nonostante queste attestazioni, raccomandiamo ai nostri lettori di evitare quest'uso, oggi considerato improprio, ristretto alle varietà regionali e popolari, e di attenersi alla norma. 
Pienamente accettabile (e vitale nell'italiano contemporaneo) è invece la costruzione causativa far imparare come sinonimo di insegnare; si veda questo contesto:

Fra gli obblighi innumerevoli, oltre alla naturale simpatia che mi legano al mio Michelotti con una catena d'oro massiccio, non è il minore quello d'avermi fatto imparare a scrivere toscanamente e con ogni possibile correttezza. (Giuseppe Baretti, Lettere familiari, XLVII, I839)

La questione sollevata, tuttavia, merita di essere ulteriormente approfondita, osservando anche quanto avviene nelle lingue europee antiche e moderne; potremo cosi anche tentare di fornire una spiegazione sull'impiego di imparare come sinonimo di insegnare. Partiamo sempre dalla nostra lingua. I due verbi fanno parte della famiglia dei verbi riguardanti l'apprendimento, i più comuni dei quali sono, da un lato, insegnare e istruire, dall'altro, imparare e apprendere.

La prima coppia fa riferimento all'azione di colui che fornisce la conoscenza, sottolineando il ruolo formatore/formativo: il verbo insegnare, che in italiano compare a partire dal secolo XIII, deriva dal lat. tardo insignāre, 'imprimere un segno'; istruire, deriva dal lat. in-struère, 'costruire dentro'. Entrambi i verbi alludono alla grande missione dell'insegnante: forgiare l'animo dei ragazzi a lui affidati, imprimendovi un segno, costruendoli per la vita.

La seconda coppia si riferisce al discente, che apprende le conoscenze elargite dall'insegnante o derivanti dalle esperienze di vita. Il verbo imparare deriva dal lat. *imparāre (composto dal prefisso con valore rafforzativo in- e parāre, 'procurarsi, acquistarsi'), e allude al fatto che con tale azione il discepolo acquista per sé la conoscenza; apprendere, introdotto nella prima metà del secolo XIII, deriva dal lat. ad-prehendère, 'afferrare', e sottolinea come chi impara afferri le conoscenze fornitegli.

Le due coppie di verbi illustrate dimostrano come le due azioni dell'insegnare e dell'apprendere siano viste come distinte in italiano. Una tale distinzione era anche marcata dalle lingue classiche, il greco e il latino. Ci limiteremo a considerare i verbi di apprendimento e le loro costruzioni più frequenti.

In greco incontriamo il verbo causativo ঠøøáøк <didáskōs, che, letteralmente, significava 'far imparare' (quindi 'insegnare'). Esso ammetteva varie costruzioni, le più frequenti delle quali erano quelle del

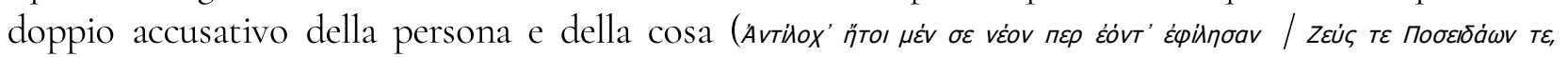

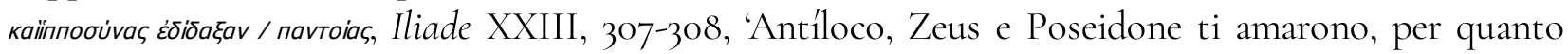
giovane, e ti insegnarono tutte le arti dei cavalieri') e quella con accusativo della persona e infinito

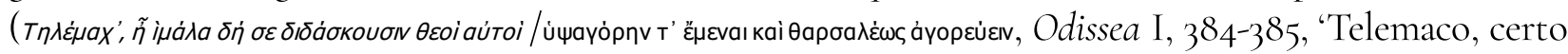
gli stessi dèi ti insegnano a fare il grande parlatore e a chiacchierare in modo temerario'). Lo stesso

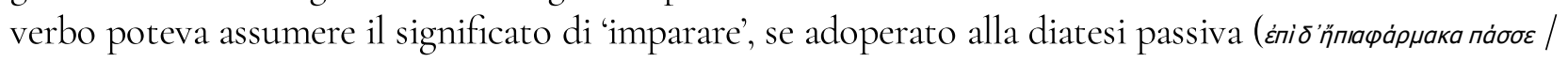

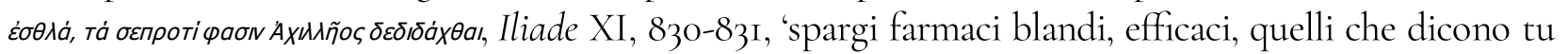
abbia imparato da Achille'). Tuttavia, in greco, per significare l'atto di imparare esisteva anche il verbo

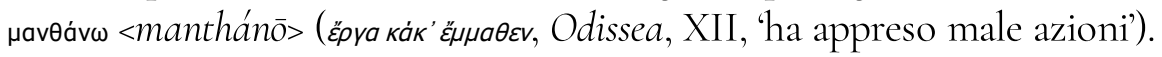

La stessa situazione si ritrova nel latino classico, dove il significato di 'insegnare' è espresso dal verbo doceo; questo si costruisce con il doppio accusativo (Te tua fata docebo, Virgilio, Eneide VI, 759, 'Ti insegnerò i tuoi destini'), o con l'accusativo e l'infinito (Tu, Tityre, lentus in umbra / formosam resonare doces Amaryllida silvas, Virgilio, Bucoliche I, 4-5, 'Tu, Titiro, tranquillo all'ombra, insegni alle selve a far risuonare il nome della bella Amarilli'). Come si è visto per la lingua greca, anche in questo caso, il verbo doceo al passivo aveva il significato di 'imparare'/apprendere': haec ab his docebantur, 'apprendevano queste cose da costoro'). In latino, il significato di 'imparare' era espresso anche da disco (disce virtutem ex me, Virgilio, Eneide XII, 435, 'impara da me la virtù'). 


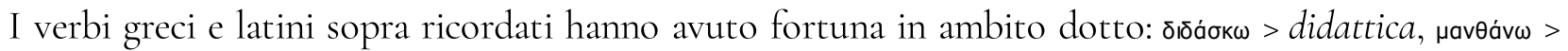
matematica (la forma di apprendimento più certa ed evidente - si ricorderà la máthesis universale cartesiana), doceo > docente, disco > discente.

La stessa distinzione di significati 'insegnare' 'imparare' si ritrova nelle lingue germaniche moderne: si ricorderà l'opposizione in inglese to teach to learn e in tedesco lehren $~$ lernen.

Nelle lingue romanze, i verbi di apprendimento sono derivati da forme latine tarde (insignare, imparare) o da forme classiche con mutamento di significato (adprehendere, 'acciuffare, afferrare').

Ritroviamo la dicotomia in spagnolo (enseñar aprender: enseñar un oración, 'insegnare una preghiera'; aprender muchas palabras, 'imparare molte parole') e in portoghese (ensinar aprender).

Più complessa è la situazione in francese: se tale lingua rende il significato di 'imparare' con il verbo apprendre (Chantal va apprendre la langue grecque, 'Chantal imparerà la lingua greca'), il significato di 'insegnare' può essere reso o con il verbo enseigner (Madame Bertin enseigne la philosophie, 'Madame Bertin insegna filosofia') o con lo stesso verbo apprendre (C'est Jean qui m’a appris à apprécier la musique classique, 'E Giovanni che mi ha insegnato ad apprezzare la musica classica'). In francese, poi, c'è un verbo "falso amico" del nostro imparare: emparer, adoperato come verbo pronominale s'emparer, che ha il significato di 'impadronirsi', 'impossessarsi' (L'ennemi s'est emparé de la ville, 'Il nemico si è impadronito della città'). Il verbo francese potrebbe anche essere all'origine del verbo italiano imparare, che in italiano antico figurava anche nella forma emparare; quando si impara, ci si impadronisce di qualcosa, conoscenza o abilità.

Anche l'uso regionale di imparare come sinonimo di insegnare potrebbe essere interpretato come una sorta di avvicinamento tipologico dell'italiano regionale centro-meridionale al francese, in cui il verbo apprendre è valido per esprimere sia l'atto di insegnare sia quello di imparare. Sull'uso improprio di imparare per insegnare avrà agito anche il sopra ricordato valore causativo di far imparare 'insegnare': se si fa attenzione, spesso, quando si usa imparare per insegnare, lo sguardo si focalizza implicitamente sul valore dell'insegnante e/o della cosa insegnata; alcuni lettori, certamente, avranno ascoltato la canzone napoletana di Dino Verde e Armando Trovajoli, Che mm'hê 'mparato a ffá? (1956), in cui un amante, triste per la partenza dell'amata straniera, si rivolge a lei dicendo (traduco in italiano): «Perché mi hai insegnato che kiss me vuol dire "ti vorrei baciare", se poi dovevi andare via?».

Infine, se con il verbo imparare si vuole focalizzare ulteriormente l'attenzione su colui che impara, si ricorre alla forma pronominale impararsi: Mi sono imparato tre poesie a memoria. Si tratta di un uso (come anche nella frase mi sono mangiato un gelato) assai frequente nel parlato, che anche le grammatiche normative ammettono, identificandolo come riflessivo indiretto o apparente oppure come transitivo pronominale: "L'azione verbale non si "riflette" direttamente sul soggetto, ma si svolge comunque a suo beneficio, nel suo interesse o per sua iniziativa; il pronome atono non rappresenta in questo caso un complemento oggetto bensi un complemento indiretto» (Serianni I989, XI, 2I).

\section{Cita come:}

Riccardo Cimaglia, Imparare e insegnare non sono la stessa cosa, "Italiano digitale", 2019, XI, 2019/4 (ottobre-dicembre)

DOI: $10.35948 / 2532-9006 / 2020.3254$ 